\title{
Paper Sheet Control Using Steerable Nips
}

\author{
Rene Sanchez*, Roberto Horowitz**, Masayoshi Tomizuka*** \\ Department of Mechanical Engineering, University of California, Berkeley, CA 94720
}

\begin{abstract}
Many high speed color printers require that sheets be accurately controlled in order to achieve a precise alignment of colors. To accomplish this goal, a steerable nips mechanism has been proposed as an actuator to propel sheets along the printer's paper path. This steerable nips mechanism allows the sheet to be precisely controlled in the longitudinal, lateral and skew directions. In this paper we develop a control strategy based on linearization by state feedback and Dynamic Surface Control (DSC) to precisely control the position of the sheet. The proposed controller is able to move the sheet from an initial to a final state under the condition that the sheet has nonzero initial and final velocities. The system model is nonlinear and subject to four nonholonomic constraints. Two of these constraints are related to the fact that the velocities perpendicular to the wheels must be zero, and the other two constraints are due to the non-slip condition.
\end{abstract}

\section{INTRODUCTION}

Some high speed color printers require that sheets be accurately positioned so that colors can be accurately placed on the sheet. To accomplish this goal a steerable nips mechanism has been proposed as an actuator. This actuator is located at the end of the copier's paper path. The steerable nips permit a more swift correction of lateral errors. This is a challenging mechatronic problem especially when sheets must move at high speeds. The steerable nips mechanism is schematically depicted in Fig. 1.

The problem of controlling paper trajectories with steerable nips is similar to the control of two-wheel robots, such as the one studied in [1]. However, the control law proposed in [1] fails to account for singularities that arise when the steering angle of the wheels approach zero. Also, in the case of the two-wheel robot, three inputs are needed to follow a reference trajectory. This is not the case with steerable nips, where four inputs are needed due to the flexibility of the paper. The system model has four inputs, the first and second inputs rotate wheels one and two respectively. The third and fourth inputs steer wheels one and two respectively. Similar to the two-wheel robot, the steerable nips mechanism is a nonholonomic system. Analytic work related to nonholonomic systems can be found in [2], [3] and [4].

The control objective considered here is to move the sheet on the plane from an initial sheet state $\underline{x}_{s}\left(t_{0}\right)$ to a final state $\underline{x}_{s}\left(t_{f}\right)$, where the state vector of the sheet is defined by $\underline{x}_{s}(t)=\left[\begin{array}{llllllll}x & \dot{x} & y & \dot{y} & \phi & \dot{\phi} & \delta & \dot{\delta}\end{array}\right]$. Generalized coordinates $x$ and $y$ corresponds to the lateral and longitudinal position of the leading left corner of the sheet. The generalized coordinate $\phi$ represents the angular position

\footnotetext{
Email: *rsanchez@me.berkeley.edu, **horowitz@me.berkeley.edu,
} ***tomizuka@me.berkeley.edu of the sheet. The generalized coordinate $\delta$ represents the amount of deformation (buckling or stretching) along the sheet, which is the difference between the distance separating points 1 and 2 , as measured along the paper $(2 b+\delta)$ and along the straight line $(2 b)$. To move the sheet from an initial state $\underline{x}_{s}\left(t_{0}\right)$ to a final state $\underline{x}_{s}\left(t_{f}\right)$, the control strategy developed in this paper uses a combination of linearization by state feedback [5] and Dynamics Surface Control (DSC) [6] techniques.

The steerable nips mechanism is a nonholonomic system and has four nonholonomic constraints, two of these constraints come from the fact that the velocities perpendicular to the wheels must be zero, and the other two constraints are due to the no-slip condition. In the steerable nips system a positive $\delta$ represents the amount buckle on a sheet. Stretching of the paper is something undesirable since it can mark or damage the paper. Also, when the paper buckles the non-slip condition is satisfied. This is not the case when the paper is stretched. To avoid stretching some buckle is added to the sheet while it is being controlled by the steerable nip mechanism. This is accomplished by having the proposed controller track the amount of buckle, $\delta$ and the buckle rate, $\dot{\delta}$. Also, rotation of the sheet is still possible when the sheet is buckled since the sheet is transversally stiff.

Results presented in this paper show that, by using the proposed control strategy, it is possible to drive the paper from an initial sheet state $\underline{x}_{s}\left(t_{0}\right)$ with nonzero initial velocity to a final sheet state $\underline{x}_{s}\left(t_{f}\right)$ also with nonzero final velocity. This was accomplished without stretching the sheet. The remainder of this paper is organized as follows. Section II will describe the nonholonomic constraints, kinematic model, and dynamic model of the steerable nips mechanism. The control strategy is derived in section III. Simulation results will be shown in section IV. Finally, conclusions and some comments regarding the control performance are stated in section $\mathrm{V}$.

\section{Kinematic And Dynamic Model of the SteERABle Nips MEChanism}

The steerable nips is illustrated in Figs. 2 - 3. The steerable nips moves a sheet on a flat surface. Figure 2 represents an initial sheet position once the two nips are in contact with the sheet. Figure 3 represents a sheet position while it is being tracked. The left corner of the sheet, point $C$, will be used to track the position of the sheet. The angular orientation of the sheet is $\phi$. Note that while the paper buckles, point $C$ remains on the flat surface since the buckle occurs only between points 1 and 2 . For this reason point $C$ does not move perpendicular to the sheet. 

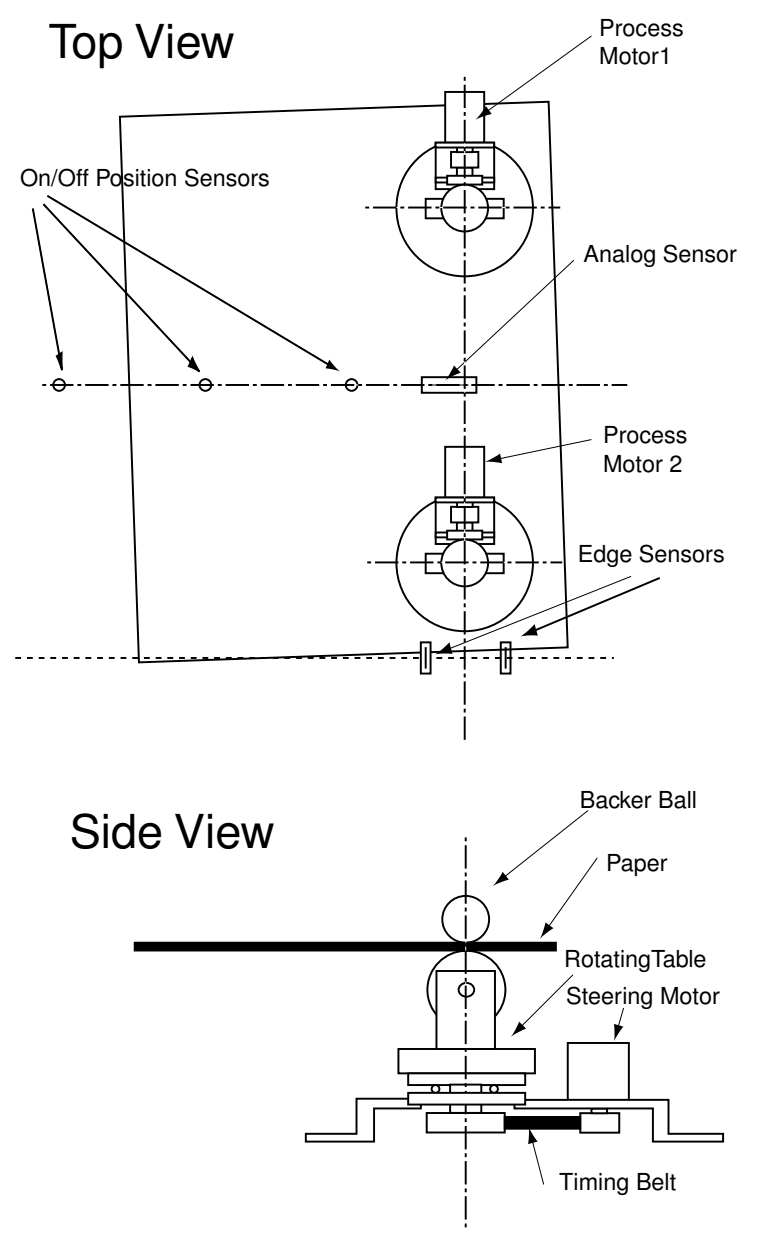

Fig. 1. Steerable Nips Shematic

It is assumed that when the sheet buckles, the sheet is still transversally stiff so rotation is possible. This is illustrated in Fig. 3 where any line perpendicular to the line that connects points 1 and 2 drawn on the buckle surface is parallel to the flat surface.

\section{A. Notation}

Figure 4 shows a schematic representation of the modeling variables for the steerable nips system. This system has two independent steering wheels, located at points 1 and 2 . These steerable wheels are separated by a distance $2 b$. Three coordinate frames are defined to describe the position and orientation of the paper: A fixed global coordinate system denoted $\left(\underline{i}_{f}, \underline{j}_{f}, \underline{k}_{f}\right)$, and two local frames $\left(\underline{i}_{1}, \underline{j}_{1}, \underline{k}_{1}\right)$ and $\left(\underline{i}_{2}, \underline{j}_{2}, \underline{k}_{2}\right)$ attached to wheel 1 and 2 respectively. The generalized coordinates of the system are $\left(x, y, \phi, \delta, \theta_{1}, \theta_{2}, \phi_{1}, \phi_{2}\right)$. Generalized coordinates $x$ and $y$ will be used to respectably represent the lateral and longitudinal position of the left corner of the sheet (point $C$ ), the generalized coordinate $\phi$ represents the angular position of the sheet and the generalized coordinate $\delta$ represents the amount of buckle of the sheet. Generalized

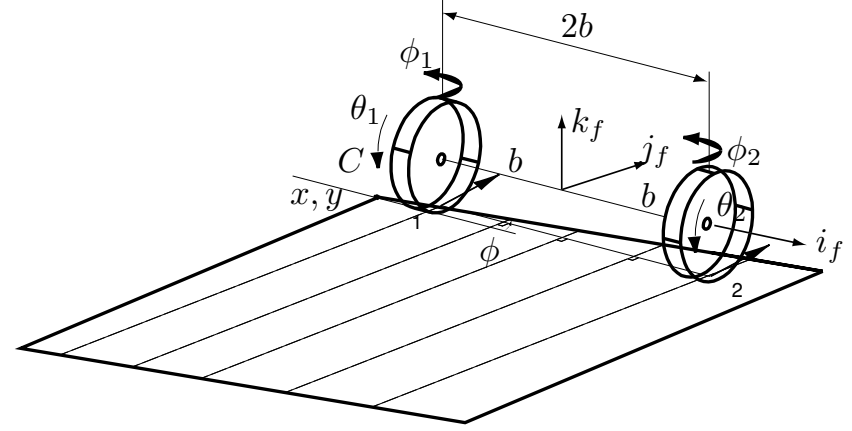

Fig. 2. Steerable Nips System

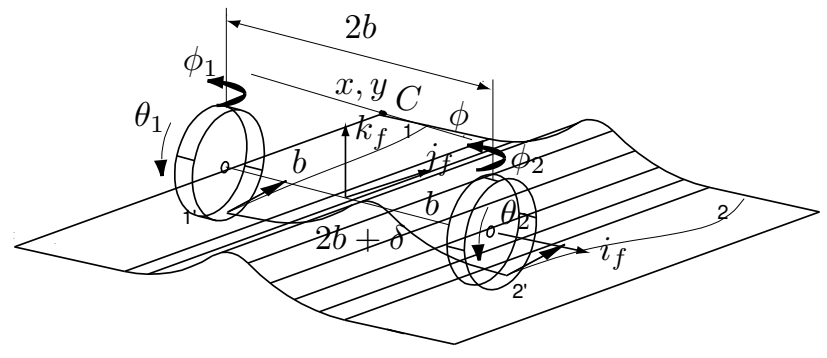

Fig. 3. Steerable Nips with Paper Buckle

coordinates $\theta_{1}$ and $\phi_{1}$ will be used to respectably describe the angular position of wheel 1 in the directions parallel and perpendicular to the sheet. Likewise, $\theta_{2}$ and $\phi_{2}$ will respectably describe the angular position of wheel 2 in the directions parallel and perpendicular to the sheet.

\section{B. Velocities}

The velocities of the paper at points 1 and 2 in global coordinates are

$$
\begin{gathered}
\underline{v}_{1}=(\dot{x}+\dot{\phi} y) \underline{i}_{f}+(\dot{y}-\dot{\phi}(x+b)) \underline{j}_{f} \\
\underline{v}_{2}=(\dot{x}+\dot{\phi} y) \underline{i}_{f}+(\dot{y}+\dot{\phi}(-x+b+\delta)) \underline{j}_{f}
\end{gathered}
$$

Invoking the non-slip condition, they can also be written in terms of the angular speed of the wheels in local coordinates:

$$
\begin{aligned}
& \underline{v}_{1}=r \dot{\theta}_{1} \underline{i}_{1} \\
& \underline{v}_{2}=r \dot{\theta}_{2} \underline{i}_{2}
\end{aligned}
$$

where $r$ is the radius of the wheels.

\section{Constraint Equations}

Four constraint equations can be obtained by writing Eq. (1) and Eq. (2) in terms of the local coordinates. That is

$$
\begin{aligned}
& \underline{v}_{1}=\left((\dot{x}+\dot{\phi} y) \cos \phi_{1}-\left(\dot{y}-\dot{\phi}(x+b) \sin \phi_{1}\right)\right) \underline{i}_{1} \\
& \quad+\left((\dot{x}+\dot{\phi} y) \sin \phi_{1}+\left(\dot{y}-\dot{\phi}(x+b) \cos \phi_{1}\right)\right) \underline{j}_{1}
\end{aligned}
$$




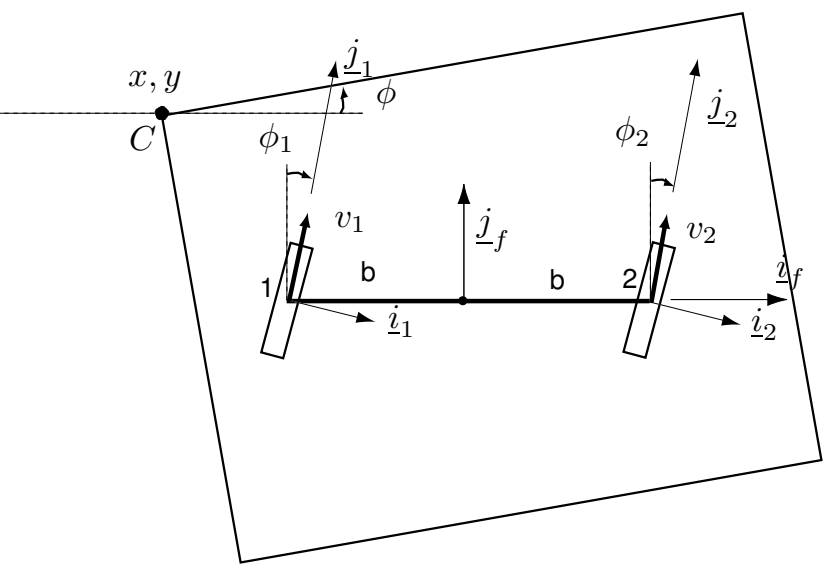

Fig. 4. Two Wheel Moving Bar

$$
\begin{aligned}
& \underline{v}_{2}=\left((\dot{x}+\dot{\phi} y) \cos \phi_{2}-\left(\dot{y}+\dot{\phi}(-x+b+\delta) \sin \phi_{2}\right)\right) \underline{i}_{2} \\
& \quad+\left((\dot{x}+\dot{\phi} y) \sin \phi_{2}+\left(\dot{y}+\dot{\phi}(-x+b+\delta) \cos \phi_{2}\right)\right) \underline{j}_{2}
\end{aligned}
$$

This provides four nonholonomic constraints. Two come from the fact that the velocities perpendicular to the wheels at points 1 and 2 are zero. This means that the velocity at wheel 1 in the direction $\underline{i}_{1}$ must be zero. The same must be true for wheel 2. The other two constraints are due to the non-slip condition. Using the previously defined generalized coordinate $p=\left[\begin{array}{llllllll}x & y & \phi & \delta & \theta_{1} & \theta_{2} & \phi_{1} & \phi_{2}\end{array}\right]^{T}$, each constraint can be written in Pfaffian form [7] as:

$$
a_{i}(p) \dot{p}=0 \quad i=1, \ldots, 4 \quad p \in \mathbb{R}^{8}
$$

For our system the constraints can be written in matrix form as

$$
A(p) \dot{p}=0
$$

where $A(p)$ is the $4 \times 8$ matrix defined below.

$$
A(p)=\left[\begin{array}{cccccccc}
a_{11} & a_{12} & a_{13} & 0 & 0 & 0 & 0 & 0 \\
a_{21} & a_{22} & a_{23} & 0 & a_{25} & 0 & 0 & 0 \\
a_{31} & a_{32} & a_{33} & a_{34} & 0 & 0 & 0 & \\
a_{41} & a_{42} & a_{43} & a_{44} & 0 & a_{46} & 0 & 0
\end{array}\right]
$$

$a_{11}=\cos \phi_{1}, a_{12}=-\sin \phi_{1}, a_{13}=y \cos \phi_{1}+$ $(x+b) \sin \phi_{1}, a_{21}=\sin \phi_{1}, a_{22}=\cos \phi_{1}, a_{23}=$ $y \sin \phi_{1}-(x+b) \cos \phi_{1}, a_{25}=-r, a_{31}=\cos \phi_{2}, a_{32}=$ $-\sin \phi_{2}, a_{33}=y \cos \phi_{2}-(-x+b+\delta) \sin \phi_{2}, a_{34}=$ $\cos \phi_{2}, a_{41}=\sin \phi_{2}, a_{42}=\cos \phi_{2}, a_{43}=y \sin \phi_{2}+(-x+$ $b+\delta) \cos \phi_{2}, a_{44}=\sin \phi_{2}, a_{46}=-r$.

These constraints are nonholonomic and therefore cannot be integrated.

\section{Kinematic Model}

The kinematic model represents the kinematic relation of the system in the direction that it can move. These are the directions of motion where the nonholonomic constraints are satisfied at all times. As detailed in [7] this is given by a basis of the right null space of the constraints, which will be denoted by $g_{j}(p) \in \mathbb{R}^{n}, j=1, \ldots, n-k=m$. By construction, this basis satisfies

$a_{i}(p) g_{j}(p)=0 \quad i=1, \ldots, k \quad j=1, \ldots, n-k \quad p \in \mathbb{R}^{n}$

and all allowable trajectories of the system can thus be written as the possible solutions of the system

$$
\dot{p}=g_{1}(p) u_{1}+\ldots+g_{m}(p) u_{m} .
$$

That is, $p(t)$ is a feasible trajectory of the system if and only if $p(t)$ satisfies Eq. (8) for a choice of control $u(t) \in \mathbb{R}^{m}$.

For our system this basis can be obtained by casting Eqs. (3) and (4) in terms of the global coordinates and equating them to Eqs. (1) and (2) respectively. This gives the following relations

$$
\begin{gathered}
\dot{x}=\left(r \sin \phi_{1}+\frac{y r}{2 b+\delta} \cos \phi_{1}\right) \dot{\theta}_{1}-\frac{y r}{2 b+\delta} \cos \phi_{2} \dot{\theta}_{2} \\
\dot{y}=\left(r \cos \phi_{1}-\frac{(x+b) r}{2 b+\delta} \cos \phi_{1}\right) \dot{\theta}_{1}+\frac{(x+b) r}{2 b+\delta} \cos \phi_{2} \dot{\theta}_{2} \\
\dot{\phi}=\frac{r}{2 b+\delta}\left(\cos \phi_{2} \dot{\theta}_{2}-\cos \phi_{1} \dot{\theta}_{1}\right) \\
\dot{\delta}=r \sin \phi_{2} \dot{\theta}_{2}-r \sin \phi_{1} \dot{\theta}_{1}
\end{gathered}
$$

The above equations are the kinematic equations of our system. They can be written in the following form

$$
\underline{\dot{p}}=G(\underline{p}) \underline{\dot{\eta}}
$$

where

$$
\underline{\dot{p}}=\left[\begin{array}{c}
\dot{x} \\
\dot{y} \\
\dot{\phi} \\
\dot{\delta} \\
\dot{\dot{\theta}_{1}} \\
\dot{\theta}_{2} \\
\dot{\phi}_{1} \\
\dot{\phi}_{2}
\end{array}\right], G(\underline{p})=\left[\begin{array}{cccc}
g_{11} & g_{12} & 0 & 0 \\
g_{21} & g_{22} & 0 & 0 \\
g_{31} & g_{32} & 0 & 0 \\
g_{41} & g_{42} & 0 & 0 \\
1 & 0 & 0 & 0 \\
0 & 1 & 0 & 0 \\
0 & 0 & 1 & 0 \\
0 & 0 & 0 & 1
\end{array}\right], \underline{\dot{\eta}}=\left[\begin{array}{c}
\dot{\theta}_{1} \\
\dot{\theta}_{2} \\
\dot{\phi}_{1} \\
\dot{\phi}_{2}
\end{array}\right]
$$

with

$g_{11}=r \sin \phi_{1}+\frac{y r}{2 b+\delta} \cos \phi_{1}, g_{12}=-\frac{y r}{2 b+\delta} \cos \phi_{2}, g_{21}=$ $r \cos \phi_{1}-\frac{(x+b) r}{2 b+\delta} \cos \phi_{1}, g_{22}=\frac{(x+b) r}{2 b+\delta} \cos \phi_{2}, g_{31}=$ $-\frac{r}{2 b+\delta} \cos \phi_{1}, g_{32}=\frac{r}{2 b+\delta} \cos \phi_{2}, g_{41}=-r \sin \phi_{1}, g_{42}=$ $r \sin \phi_{2}$

In the above equation $\dot{\eta} \in \mathbb{R}^{4}$ is a vector of independent velocities. Note that, in general, $\dot{\eta}$ is smooth in $p$, and linear in $\underline{\dot{p}},(\dot{\underline{\eta}}(\underline{p}, \underline{\dot{p}}))$ [3]. The above equation indicates that velocities $\dot{\theta}_{1}, \dot{\theta}_{2}, \dot{\phi}_{1}, \dot{\phi}_{2}$ are sufficient to determine the instantaneous velocities of all generalized coordinates of the system. Also, note that the velocities calculated with Eq.(13) satisfy the nonholonomic constraints, since $G(\underline{p})=\left[\begin{array}{llll}g_{1}(\underline{p}) & g_{2}(\underline{p}) & g_{3}(\underline{p}) & g_{4}(\underline{p})\end{array}\right]$ is the right null space of the constraints. That is

$$
A(\underline{p}) g_{j}(\underline{p})=0
$$

Equation (13) is referred to as the kinematic state-model [2]. 


\section{E. Dynamic Model}

A dynamic model of the system is obtained by considering only the dynamics of the actuators and neglecting the inertia of the sheet. For simplicity, we consider actuator dynamics of the following form

$$
J \underline{\ddot{\eta}}=\underline{u}
$$

where

$$
J=\left[\begin{array}{llll}
1 & 0 & 0 & 0 \\
0 & 1 & 0 & 0 \\
0 & 0 & 1 & 0 \\
0 & 0 & 0 & 1
\end{array}\right], \ddot{\eta}=\left[\begin{array}{c}
\ddot{\theta}_{1} \\
\ddot{\theta}_{2} \\
\ddot{\phi}_{1} \\
\ddot{\phi}_{2}
\end{array}\right], \underline{u}=\left[\begin{array}{l}
u_{1} \\
u_{2} \\
u_{3} \\
u_{4}
\end{array}\right]
$$

Making use of kinematic state-model derived in section IID. The time derivative of Eq. (13) is

$$
\underline{\ddot{p}}=\frac{\partial}{\partial \underline{p}}[G \underline{\dot{\eta}}] \underline{\dot{p}}+G \underline{\ddot{\eta}}
$$

Combining Eq. (14) and Eq. (15) we obtain

$$
\underline{\ddot{p}}=C(\underline{p}, \underline{\dot{p}})+B(\underline{p}) \underline{u}
$$

In Eq. (16), $C(\underline{p}, \underline{\dot{p}})$ and $B(\underline{p})$ are computed as:

$$
C(\underline{p}, \underline{\dot{p}})=\frac{\partial}{\partial \underline{p}}[G \underline{\dot{\eta}}] \underline{\dot{p}}, B(\underline{p})=G(\underline{p}) J^{-1}
$$

where

$$
C(\underline{p}, \underline{\dot{p}})=\left[\begin{array}{c}
c_{1} \\
c_{2} \\
c_{3} \\
c_{4} \\
0 \\
0 \\
0 \\
0
\end{array}\right], B(\underline{p})=\left[\begin{array}{cccc}
b_{11} & b_{12} & 0 & 0 \\
b_{21} & b_{22} & 0 & 0 \\
b_{31} & b_{32} & 0 & 0 \\
b_{41} & b_{42} & 0 & 0 \\
1 & 0 & 0 & 0 \\
0 & 1 & 0 & 0 \\
0 & 0 & 1 & 0 \\
0 & 0 & 0 & 1
\end{array}\right]
$$

$c_{1}=-\dot{\phi} \dot{y}+\frac{y \dot{\delta} \dot{\phi}}{2 b+\delta}+\left(r \cos \phi_{1} \dot{\theta}_{1}-\frac{y r}{2 b+\delta} \sin \phi_{1} \dot{\theta}_{1}\right) \dot{\phi}_{1}+$ $\frac{y r}{2 b+\delta} \sin \phi_{2} \dot{\theta}_{2} \dot{\phi}_{2}, c_{2}=\dot{\phi} \dot{x}-\frac{(x+b) \dot{\delta} \dot{\phi}}{2 b+\delta}+\left(-r \sin \phi_{1} \dot{\theta}_{1}+\right.$ $\left.\frac{(x+b) r}{2 b+\delta} \sin \phi_{1} \dot{\theta}_{1}\right) \dot{\phi}_{1}-\frac{(x+b) r}{2 b+\delta} \sin \phi_{2} \dot{\theta}_{2} \dot{\phi}_{2}, c_{3}=-\frac{\dot{\delta} \dot{\phi}}{2 b+\delta}+$ $\frac{r}{2 b+\delta} \sin \phi_{1} \dot{\theta}_{1} \dot{\phi}_{1}-\frac{r}{2 b+\delta} \sin \phi_{2} \dot{\theta}_{2} \dot{\phi}_{2}, c_{4}=-r \cos \phi_{1} \dot{\theta}_{1} \dot{\phi}_{1}+$ $r \cos \phi_{2} \dot{\theta}_{2} \dot{\phi}_{2}, b_{11} \stackrel{r}{=} r \sin \phi_{1}+\frac{y r}{2 b+\delta} \cos \phi_{1}, b_{12}=$ $-\frac{y r}{2 b+\delta} \cos \phi_{2}, b_{21}=r \cos \phi_{1}-\frac{(x+b) r}{2 b+\delta} \cos \phi_{1}, b_{22}=$ $\frac{(x+b) r}{2 b+\delta} \cos \phi_{2}, b_{31}=-\frac{r}{2 b+\delta} \cos \phi_{1}, b_{32}=$ $\frac{r}{2 b+\delta} \cos \phi_{2}, b_{41}=-r \sin \phi_{1}, b_{42}=r \sin \phi_{2}$.

Equation (16) is the dynamic model of the steerable nips with a sheet allowed to buckled.

This system can be represented in state space form by choosing the following state vector $\underline{x}=$ $\left[\begin{array}{llllllllllllllll}x & y & \phi & \delta & \theta_{1} & \theta_{2} & \phi_{1} & \phi_{2} & \dot{x} & \dot{y} & \dot{\phi} & \dot{\delta} & \dot{\theta}_{1} & \dot{\theta}_{2} & \dot{\phi}_{1} & \dot{\phi}_{2}\end{array}\right]^{T}$. This gives

$$
\underline{\dot{x}}=\left[\begin{array}{c}
\dot{\dot{p}} \\
C(\underline{\underline{p}}, \underline{\dot{p}})
\end{array}\right]+\left[\begin{array}{c}
0^{8 \times 4} \\
B(\underline{p})
\end{array}\right] \underline{u}
$$

\section{Feedback Control}

In this section we derive the control law. This derivation makes use of linearization by state feedback [8] and Dynamic Surface Control (DSC) [6]. The following is the nonlinear system derived in the previous section

$$
\begin{aligned}
& \underline{\dot{x}}=\left[\begin{array}{c}
\dot{\dot{p}} \\
C(\underline{\underline{p}}, \underline{\dot{p}})
\end{array}\right]+\left[\begin{array}{c}
0^{8 \times 4} \\
B(\underline{p})
\end{array}\right] \underline{u} \\
& \underline{y}=\underline{h}(\underline{x})=\left[\begin{array}{l}
x \\
y \\
\phi \\
\delta
\end{array}\right]
\end{aligned}
$$

The above system is a square Multi-Input Multi-Output (MIMO) system. It is a square system since it has as many inputs as outputs. Differentiating the output function with respect to time, as described in [5], Eq. (19) is obtained. Note that Eq. (19) does not have inputs $u_{3}$ and $u_{4}$. These inputs are accessed by using a technique similar to Dynamic Surface Control (DSC). That is, we make $\dot{\phi}_{1}$, and $\dot{\phi}_{2}$ to be pseudo inputs.

$$
\underline{\ddot{y}}=M(\underline{x})+N(\underline{x})\left[\begin{array}{c}
u_{1} \\
u_{2} \\
\dot{\bar{\phi}}_{1 d} \\
\dot{\bar{\phi}}_{2 d}
\end{array}\right]
$$

$\dot{\bar{\phi}}_{1 d}$ and $\dot{\bar{\phi}}_{2 d}$ are the desired values of $\dot{\phi}_{1}$ and $\dot{\phi}_{2}$ (synthetic inputs). The matrix $M(\underline{x})$ and $N(\underline{x})$ are computed as follow

$$
M(\underline{x})=\left[\begin{array}{c}
m_{1} \\
m_{2} \\
m_{3} \\
0
\end{array}\right], N(\underline{x})=\left[\begin{array}{llll}
n_{11} & n_{12} & n_{13} & n_{34} \\
n_{21} & n_{22} & n_{23} & n_{24} \\
n_{31} & n_{32} & n_{33} & n_{34} \\
n_{41} & n_{42} & n_{43} & n_{44}
\end{array}\right]
$$

$m_{1}=-\dot{\phi} \dot{y}+\frac{y \dot{\delta} \dot{\phi}}{2 b+\delta}, m_{2}=\dot{\phi} \dot{x}-\frac{(x+b) \dot{\delta} \dot{\phi}}{2 b+\delta}, m_{3}=$ $-\frac{\dot{\delta} \dot{\phi}}{2 b+\delta}, n_{11}=\frac{y r}{2 b+\delta} \cos \phi_{1}+r \sin \phi_{1}, n_{12}=$ $-\frac{y r}{2 b+\delta} \cos \phi_{2}, n_{13}=r \cos \phi_{1} \dot{\theta}_{1}-\frac{y r}{2 b+\delta} \sin \phi_{1} \dot{\theta}_{1}, n_{14}=$ $\frac{y r}{2 b+\delta} \sin \phi_{2} \dot{\theta}_{2}, n_{21}=r \cos \phi_{1}-\frac{(x+b) r}{2 b+\delta} \cos \phi_{1}, n_{22}=$ $\frac{(x+b) r}{2 b+\delta} \cos \phi_{2}, n_{23}=-r \sin \phi_{1} \dot{\theta}_{1}+\frac{(x+b) r}{2 b+\delta} \sin \phi_{1} \dot{\theta}_{1}, n_{24}=$ $-\frac{(x+b) r}{2 b+\delta} \sin \phi_{2} \dot{\theta}_{2}, n_{31}=-\frac{r}{2 b+\delta} \cos \phi_{1}, n_{32}=$ $\frac{r}{2 b+\delta} \cos \phi_{2}, n_{33}=\frac{r}{2 b+\delta} \sin \phi_{1} \dot{\theta}_{1}, n_{34}=$ $-\frac{r}{2 b+\delta} \sin \phi_{2} \dot{\theta}_{2}, n_{41}=-r \sin \phi_{1}, \quad n_{42}=r \sin \phi_{2}, n_{43}=$ $-r \cos \phi_{1} \dot{\theta}_{1}, n_{44}=r \cos \phi_{2} \dot{\theta}_{2}$.

Then by choosing the following state feedback law

$$
\left[\begin{array}{c}
u_{1} \\
u_{2} \\
\dot{\bar{\phi}}_{1 d} \\
\dot{\bar{\phi}}_{2 d}
\end{array}\right]=N^{-1}(\underline{x})(\underline{v}-M(\underline{x}))
$$

where

$$
N^{-1}(x)=\left[\begin{array}{cccc}
i n_{11} & i n_{12} & i n_{13} & 0 \\
i n_{21} & i n_{22} & i n_{23} & i n_{24} \\
i n_{31} & i n_{32} & i n_{33} & 0 \\
i n_{41} & i n_{42} & i n_{43} & i n_{44}
\end{array}\right]
$$


with

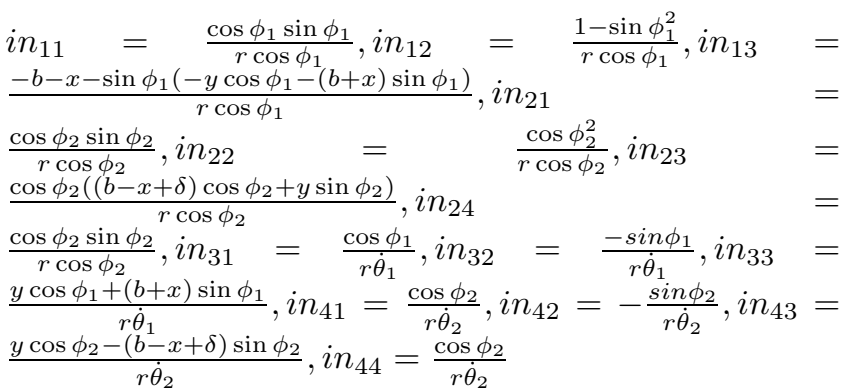

the linear close loop system results:

$$
\left[\begin{array}{c}
\ddot{x} \\
\ddot{y} \\
\ddot{\phi} \\
\ddot{\delta}
\end{array}\right]=\left[\begin{array}{l}
v_{1} \\
v_{2} \\
v_{3} \\
v_{4}
\end{array}\right]
$$

At this points we have four decoupled equations. This means that $v_{1}, v_{2}, v_{3}, v_{4}$ only affect the outputs $x, y, \phi, \delta$ respectably. Choose $v_{1}=\ddot{x}_{d}+k_{1} \dot{\tilde{x}}+q_{1} \tilde{x}, v_{2}=\ddot{y}_{d}+k_{2} \dot{\tilde{y}}+$ $q_{2} \tilde{y}, v_{3}=\ddot{\phi}_{d}+k_{3} \dot{\tilde{\phi}}+q_{3} \tilde{\phi}$ and $v_{4}=\ddot{\delta}_{d}+k_{4} \dot{\tilde{\delta}}+q_{4} \tilde{\delta}$ where $\tilde{x}=x_{d}-x, \tilde{y}=y_{d}-y, \tilde{\phi}=\phi_{d}-\phi, \tilde{\delta}=\delta_{d}-\delta$, and $k_{1}, k_{2}, k_{3}, k_{4}, q_{1}, q_{2}, q_{3}, q_{4}$ are positive constants. The choice of $v_{i}$ with positive constants for $k_{i}, q_{i}$ gives exponentially decaying errors. The differential equations of these errors will be $\ddot{\tilde{x}}_{i}+k_{1} \dot{\tilde{x}}+q_{1} \tilde{x}=0, \ddot{\tilde{y}}_{i}+k_{2} \dot{\tilde{x}}+q_{2} \tilde{x}=0$, $\tilde{\phi}_{i}+k_{3} \dot{\tilde{\phi}}+q_{3} \tilde{\phi}=0$ and $\tilde{\delta}_{i}+k_{4} \tilde{\tilde{\delta}}+q_{4} \tilde{\delta}=0$.

$\dot{\bar{\phi}}_{1}$ and $\dot{\bar{\phi}}_{2}$ are not actual inputs but rather the desired values of $\phi_{1}$ and $\phi_{2}$. In our case we have access to $\ddot{\phi}_{1}=u_{3}$ and $\ddot{\phi}_{2}=u_{4}$ and not to $\dot{\phi}_{1}$ and $\dot{\phi}_{2}$. This problem is solved by Dynamic Surface control (DSC) technique [6]; this method is a "synthetic input technique", which is similar to backstepping [9] and multiple surface control [6] methods. The DSC method utilizes additional low pass filters, in order to overcome the problem of finding derivatives of the trajectories $\dot{\phi}_{1 d}$ and $\dot{\phi}_{2 d}$. The design procedure for determining the inputs $u_{3}$ and $u_{4}$ is as follows. Define the tracking errors between the desired and the actual angular velocities by

$$
S_{1}=\dot{\phi}_{d 1}-\dot{\phi}_{1}, \quad S_{2}=\dot{\phi}_{d 2}-\dot{\phi}_{2}
$$

and their respective derivatives by

$$
\dot{S}_{1}=\ddot{\phi}_{d 1}-u_{3}, \quad \dot{S}_{1}=\ddot{\phi}_{d 1}-u_{4}
$$

If $\dot{\phi}_{1}$ and $\dot{\phi}_{2}$ were to track $\dot{\phi}_{1 d}$ and $\dot{\phi}_{2 d}$ respectably, then $S_{1}$ and $S_{2}$ would converge to a neighborhood of 0 . $\bar{\phi}_{1}$ and $\bar{\phi}_{1}$ which are derived in Eq. (20) are passed through first order filters, i.e.

$$
\begin{array}{ll}
\tau_{1} \ddot{\phi}_{1 d}+\dot{\phi}_{1 d}=\dot{\bar{\phi}}_{1}, & \dot{\phi}_{1 d}(0)=\dot{\bar{\phi}}_{1}(0) \\
\tau_{2} \ddot{\phi}_{2 d}+\dot{\phi}_{2 d}=\dot{\bar{\phi}}_{2}, & \dot{\phi}_{2 d}(0)=\dot{\bar{\phi}}_{2}(0)
\end{array}
$$

By differentiation of $\dot{\phi}_{1 d}$ and $\dot{\phi}_{2 d}$ it is now possible to define $u_{3}$ and $u_{4}$ so as to respectively drive $S_{1}$ and $S_{2}$ to zero.

$$
\begin{aligned}
& u_{3}=\ddot{\phi}_{1 d}+C_{1} S_{1}=\frac{\dot{\bar{\phi}}_{1}-\dot{\phi}_{1 d}}{\tau_{1}}+C_{1} S_{1} \\
& u_{4}=\ddot{\phi}_{2 d}+C_{1} S_{2}=\frac{\dot{\bar{\phi}}_{2}-\dot{\phi}_{2 d}}{\tau_{2}}+C_{2} S_{2}
\end{aligned}
$$

This choice of inputs force the errors $S_{1}$ and $S_{2}$ to asymptotically decay to zero since

$$
\dot{S}_{2}+C_{2} S_{2}=0, \quad \dot{S}_{2}+C_{2} S_{2}=0 .
$$

where $C_{1}$ and $C_{2}$ are positive constants.

\section{Simulation Results}

The model was simulated for the following sheet initial conditions: $x\left(t_{0}\right)=-10 \mathrm{~mm}, \dot{x}\left(t_{0}\right)=$ $500 \mathrm{~mm} / \mathrm{sec}, y\left(t_{0}\right)=10 \mathrm{~mm}, \phi\left(t_{0}\right)=10^{\circ}$. Simulation results are shown in Figs. 5 - 8.

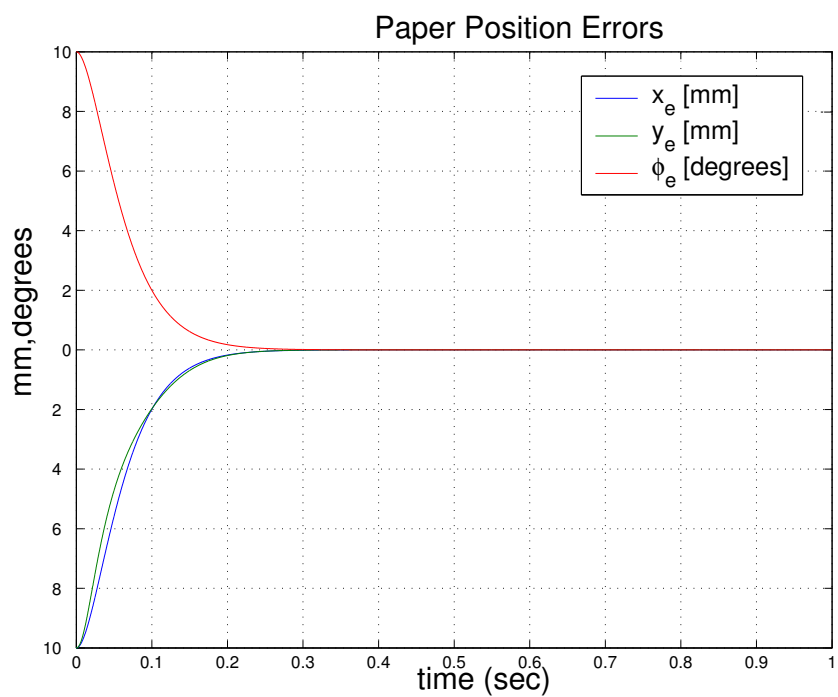

Fig. 5. Paper Position

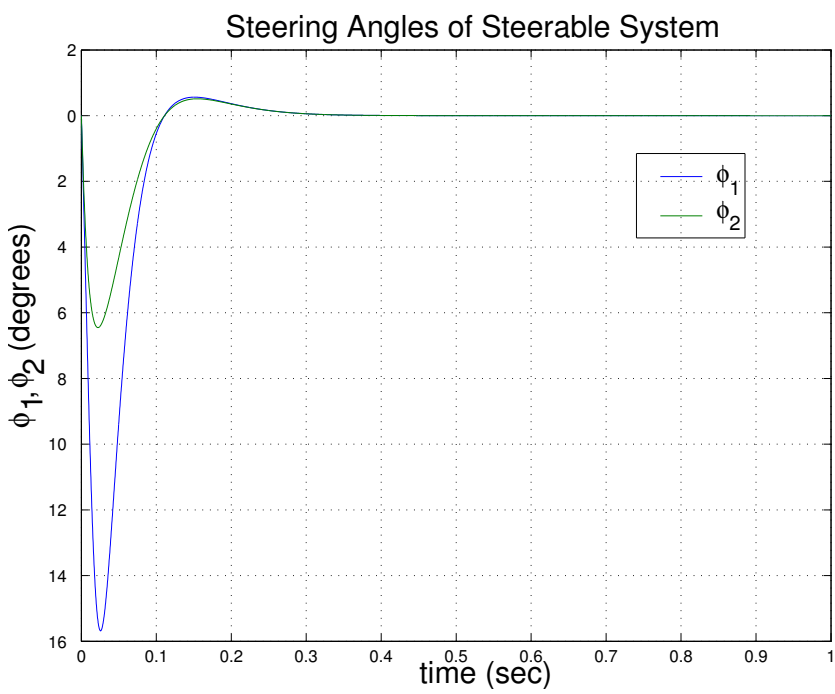

Fig. 6. Wheels Steering Angle vs. Time 


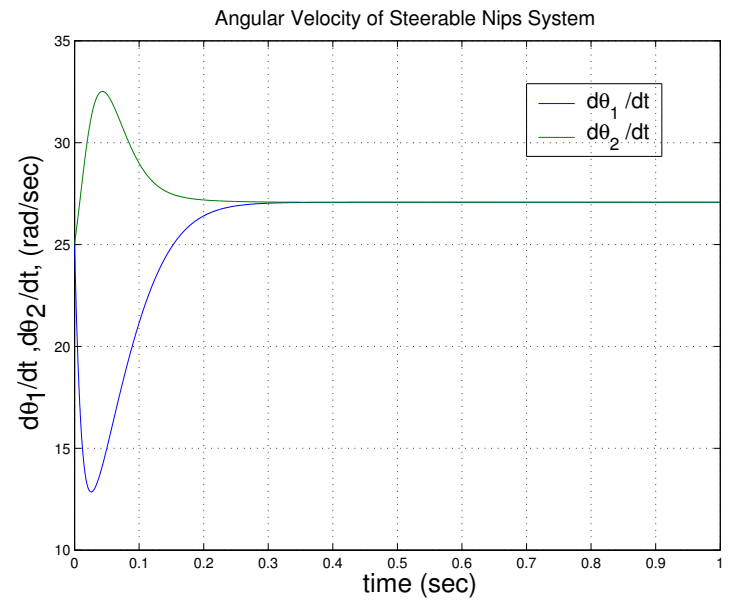

Fig. 7. Angular velocities of the Wheels vs. Time
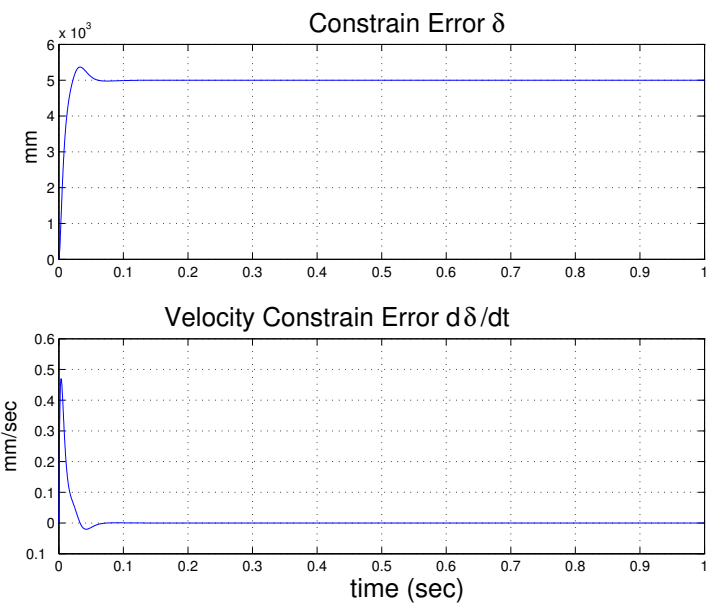

Fig. 8. Constraint $\delta$ vs. Time

Figure 5 shows the position errors of the paper as it goes from its initial state at $(x, y, \phi, \delta)=$ $\left(-12 \mathrm{~mm}, 10 \mathrm{~mm}, 10^{\circ}, 0\right)$ to its final state at $(x, y, \phi, \delta)=$ $(200 \mathrm{~mm}, 0,0,5 \mu \mathrm{m})$. The plant parameters used in this example are $b=100 \mathrm{~mm}$ and $r=20 \mathrm{~mm}$. The control parameters are $k_{1}=k_{2}=k_{3}=60, k_{4}=600, q 1=q 2=$ $q 3=900, q 4=90000, \tau_{1}=\tau_{2}=0.0001, C_{1}=C_{2}=$ 1000. Note that $C_{1}$ and $C_{2}$ are chosen so that the actuation errors from DSC decay faster than the paper errors, that is the bandwidth of actuator are greater than of the bandwidth of the control. The steering angles of both wheels are shown in Fig. 6 and the velocity of both wheels are shown in Fig. 7. The initial steering angles of the wheels are zero. They are steered immediately once the controler senses the arrival of the sheet and both wheels are in contact with the sheet. This action will correct the lateral errors. The steering angle will become zero once the lateral errors have been corrected as it is shown in Fig. 6. Also, the angular velocities of the wheels will be equal, once the angular position of the paper has been corrected, as shown in Fig. 7. Figure 8 shows the amount of buckle that the sheet has. The controller has a reference of $5 \mu \mathrm{m}$ for sheet buckle, that is $\delta_{d}=5 \mu \mathrm{m}$. This is done to avoid stretching the sheet. Since the paper is not easily stretch any negative value for $\delta$ will mark or break the paper. Also, while the paper is been actuated, any disturbance will not induce any stretching of the sheet since the sheet is already buckled a small amount.

\section{CONCLUSION}

Results obtained in this paper have shown that, by using the proposed control strategy, it is possible to drive a sheet from an initial state with nonzero longitudinal velocity to a final state also with nonzero longitudinal velocity. This proposed controller uses linearization by state feedback [5] and Dynamic Surface Control (DSC) [6] to fully control the position of a sheet from an initial state $\underline{x}_{s}\left(t_{0}\right)$ with nonzero paper velocity to a final state $\underline{x}_{s}\left(t_{f}\right)$ also with nonzero paper velocity. This is accomplished with a small amount of buckle on the sheet. The amount of sheet buckle is less than $6 \mu \mathrm{m}$. Since the induced buckle was $5 \mu \mathrm{m}$ the performance of the controller is excellent. Buckle is introduced in order to avoid stretching the sheet while it is being propel. Stretching of a sheet is not desirable since this can mark or break the sheet.

\section{ACKNOWLEDGEMENTS}

This work was support by the National Science Fundation under Award ID 0301719. The authors thank Gabriel Gomes for his numerous critical remarks and suggestions during the preparation of this manuscript.

\section{REFERENCES}

[1] Xiaoping Yun and N. Sarkar. Dynamic feedback control of vehicles with two steerable wheels. In 1996 IEEE International Conference on Robotics and Automation, pages 3105-3110, 1996.

[2] G. Campion, d'Andrea Novel, and G. Bastin. Controllability and state feedback stabilisability of nonholonomic mechanical systems. In Advanced robot control : proceedings of the International Workshop on Nonlinear and Adaptive Control: Issues in Robotics, pages 106 -124 , Grenoble, November 1990.

[3] G. Campion, B. d'Andrea Novel, and G. Bastin. Modelling and state feedback control of nonholonomic mechanical systems. In Proceedings of the 30th IEEE Conference on Decision and Control, pages 1184-1189, Brighton, England, December 1991.

[4] B. d'Andrea Novel, G. Bastin, and G. Campion. Modelling and control of non-holonomic wheeled mobile robots. In Proceedings of the 1991 IEEE Conference on Robotics and Automation, pages $1130-1135$, Sacramento, CA, April 1991.

[5] S. S. Sastry. Nonlinear Systems : Analysis, Stability, and Control. Springer, 1999.

[6] D. Swaroop, J. C. Gerdes, P. P. Yip, and J. K. Hedrick. Dynamic surface control of nonlinear systems. In Proceedings of the American Control Conference, pages 3028 -3034, Albuquerque, New Mexico, June 1997.

[7] Richard M. Murray, Zexiang Li, and Shankar S. Sastry. A Mathematical Introduction to Robotic Manipulation. CRC Press, 1993.

[8] J.-J. E. Slotine and W. Li. Applied Nonlinear Control. Prentice Hall, Inc., N.J., 1991.

[9] P. Kokotovic. The joy of feedback: nonlinear and adaptive. Control Systems Magazine, IEEE, pages 7-17, June 1991. 